\title{
Internet, biblioteca sonora: análisis, tratamiento e integración de recursos auditivos para el aula de ELE
}

\author{
Eva ÁLVAREZ RAMOS \\ Universidad de Valladolid \\ Centro Internacional de Lexicografía \\ alvarez.ramos.eva@gmail.com
}

Recibido: septiembre 2013

Aceptado: julio 2014

\section{RESUMEN}

En este trabajo, tras un breve estudio y análisis del estado de la cuestión, nos proponemos revisar una serie de materiales destinados al aprendizaje y desarrollo de la competencia auditiva en la enseñanza del español como lengua extranjera. Prestaremos especial atención a los recursos orales empleados. Revisaremos las publicaciones destinadas a desarrollar esta destreza. Examinaremos las herramientas de aprendizaje disponibles en la red con la intención de crear un breve corpus significativo que consiga poner en evidencia cuáles son las carencias más reiteradas que presentan los discursos orales en esta disciplina.

Palabras clave: textos orales, actividades comunicativas, competencia auditiva, español como lengua extranjera

Internet, audio library: Analysis, use and integration of audio resources in teaching Spanish as a foreign language

\section{ABSTRACT}

In this article, after a short survey and analysis of the state of the matter, we review some materials for learning Spanish as a foreign language, especially for the development of skill of listening, paying attention to oral resources. We revise the publications that help to develop this skill, and examine the learning tools available on the Internet, with the intention to create a significant corpus, that shed light to the most important deficiencies that the oral discourses have in this discipline.

Keywords: oral texts, communicative activities, skill of listening, Spanish as a foreign language.

Internet, bibliothèque sonore: Analyse, utilisation et intégration du ressources auditifs dans l'enseignement de l'espagnol comme langue

\section{RÉSUMÉ}

$$
\text { étrangère }
$$

Dans ce travail, après une courte enquête et une analyse de la situation, nous nous proposons d'examiner un certain nombre de matériaux pour l'apprentissage et le développement de la compétence auditive en l'enseignement de l'espagnol comme langue 
étrangère. Nous porterons une attention particulière aux ressources orales utilisées. Nous réviserons les publications destinées à développer cette compétence. Nous examinerons les outils d'apprentissage disponibles sur le web avec l'intention de créer un corpus important qui réussisse à mettre en évidence les manques les plus réitérés qui présentent les discours oraux dans cette discipline.

Mots-clés: textes oraux, activités de communication, compétence auditive, l'espagnol comme langue étrangère

SUMARIO: 1. Algunas nociones básicas. 2. Discursos orales tradicionales. 3. La comprensión auditiva de hablas reales. 4. Aplicaciones didácticas. 5. Conclusiones. 6. Bibliografía.

\section{ALGUNAS NOCIONES BÁSICAS}

El Diccionario de términos clave de ELE ${ }^{1}$ (Martín Peris, 2008) hace referencia a la comprensión auditiva (CA) como a una destreza lingüística, aquella que se refiere a la interpretación del discurso oral. En los procesos de comprensión verbal intervienen muchos factores: no solo necesitamos unos saberes únicamente lingüísticos, sino que elementos como los pragmáticos y sociológicos o los cognitivos y de actitud son estrictamente necesarios para que la intelección llegue a buen puerto. Así esta capacidad comunicativa,

abarca el proceso completo de interpretación del discurso, desde la mera descodificación y comprensión lingüística de la cadena fónica (fonemas, sílabas, palabras, etc.) hasta la interpretación y la valoración personal; de modo que, a pesar de su carácter receptivo, requiere una participación activa del oyente. (DTC)

Los procedimientos de descodificación se llevan a cabo desde dos perspectivas opuestas pero no por ello excluyentes ${ }^{2}$. Contamos con la teoría defendida por Koster (1991) que, basándose en procedimientos sintéticos, describe el engranaje a modo de mecano lingüístico y cómo el aprendiente va desgranando y articulando por orden creciente ${ }^{3}$ los niveles fonológicos, léxicos, morfosintácticos, semánticos hasta acceder finalmente al nivel pragmático.

\footnotetext{
${ }^{1}$ DTC en adelante.

${ }^{2}$ Cook señala que los hablantes (independientemente de que la lengua a comprender sea materna o meta) utilizan indistinta y simultáneamente las técnicas sintéticas y analíticas en los procesos de comprensión auditiva para conseguir así una eficiencia máxima cognoscitiva (Cook, 2004, 128).

${ }^{3}$ Rodríguez Abella especifica que el modelo defendido por Koster no es tan jerárquico como pudiera parecer, la organización está más cercana a la heterarquía, es decir, todos los componentes son independientes, influyen unos sobre los otros y se relacionan entre sí de manera bidireccional (Rodríguez Abella, 2002, 237).
} 
Del mismo modo podemos proceder a la inversa y acceder al significado en un movimiento descodificador, que nos lleve del todo generalizador a la parte más mínima individualizada; es lo que se conoce como procesamiento analítico. Se comienza "por los aspectos más generales del discurso - el tipo de texto, el conocimiento de la situación, la idea general de cada párrafo oral, etc.posteriormente el oyente entra en los pormenores y matices de las unidades lingüísticas menores" (DTC).

La experiencia en el aula demuestra que ambos procesos son constituyentes de la descodificación final y que los alumnos emplean ambos para conseguir así el fin buscado; por este motivo el docente no debe pasar por alto la necesidad intrínseca de adaptar su enseñanza a los procesos de aprendizaje, "un modelo que dé cuenta del funcionamiento de la comprensión auditiva debe ser global y considerar que existe una interrelación íntima y constante entre los diversos niveles lingüísticos que componen el enunciado" (Poch Olivé, 1988, 14).

Independientemente del proceso o los procesos que se activen en el aula para optimizar los procedimientos interpretativos, el docente ha de tener, asimismo, en cuenta todos los factores del hecho comunicativo que afectan de forma directa a las prácticas de comprensión

...no es una destreza, sino una serie de destrezas marcadas por el hecho de involucrar la percepción auditiva de signos orales [además]...no es pasiva. Una persona puede oír algo, pero no estar escuchando... es absolutamente necesaria para cualquier otra labor que se realice con el lenguaje, especialmente para poder hablar y aún para poder escribir. (James, 1984, 3)

No solo escuchando se llega al entendimiento, hay que poner a trabajar otro tipo de procesos mentales, que nos lleven a comprender lo que se nos está diciendo; escuchar concentrados para, en una primera fase, descifrar el mensaje y en un segundo paso poder interpretarlo. Se oye mucho, se escucha poco. Es habitual protegerse de la agresión acústica externa, inconscientemente nos evadimos y desatendemos los sonidos que pueblan el mundo que nos rodea.

El acto de escuchar no es un acto pasivo, como se le ha venido calificando a lo largo del tiempo, requiere un esfuerzo y un querer escuchar, requiere que el alumno active una serie de procesos mentales que le lleven, entre otras cosas, a reconocer cuál es el objetivo del discurso que está escuchando, que le lleven a ser capaz de distinguir y separar las ideas principales de las secundarias, asimismo deberá poder seleccionar también, los términos importantes y desechar los de relleno, tampoco debe dejar de lado las implicaturas, y las inferencias de todo aquello que se encuentra implícito en el discurso. Si a esto le añadimos el reconocimiento de los 
dobles sentidos, los juegos de palabras, las ironías y sentidos figurados... ${ }^{4}$ mediten y digan si un trabajo como el anterior no requiere esfuerzo y comportamiento activo.

El modelo pedagógico que ha de primar en la destreza auditiva ha de ser el interactivo, aquel que considera el proceso de la comprensión como un camino multidireccional donde además de comprender el mensaje hay que poseer una básica competencia cultural y sociolingüística así como una serie de estrategias para recibir y posteriormente emitir mensajes (Bonch-Bruevich, 2006).

Considerar pues la CA como una destreza pasiva no responde en absoluto a la realidad (Martín Peris, 1991). Además la CA como competencia inscrita en un marco conversacional requiere una actitud activa del hablante. En toda conversación los interlocutores han de mostrar siempre interés mediante la repetición de las palabras más importantes o claves, la producción de sonidos no verbales, las afirmaciones de apoyo etc.; se colabora con el interlocutor, se le apoya asintiendo en su discurso, se le anima... Es un proceso de comunicación verbal y no verbal siguiendo el modelo de Sperber y Wilson (1994).

En el aula de español para extranjeros el oyente no desempeña ningún rol en la conversación ${ }^{5}$ y los audios grabados no permiten la interacción del estudiante que necesitará después en su día a día cotidiano. Esto trae consigo que el estudiante no desarrolle algunas de las microdestrezas necesarias en todo proceso comunicativo que busque la efectividad.

La comprensión auditiva no puede basarse, entonces, en la escucha repetida de ciertas estructuras modelo porque en la vida real al alumno se le van a exigir otro tipo de destrezas, tendrá que cubrir otras necesidades. En una conversación en la calle no recibirá las frases como pildoritas, ordenadas, bien pronunciadas, manteniendo la estructura estándar sino que se encontrará con giros populares, frases hechas, oraciones cortadas y muchos dobles sentidos.

Dunkel (1990), Long (1987) y Mueller (1980), entre otros, ${ }^{6}$ reconocen el poco tiempo que se le destina al ejercitamiento de esta destreza frente a la práctica que se hace de la escritura, la lectura y la conversación ${ }^{7}$. Algo que resulta paradójico si

${ }^{4}$ Para un estudio más detallado de las habilidades de la escucha puede consultarse Rost (1991).

5 Juan Carlos Casañ Núñez afirma que "es necesario otorgar un rol y un propósito al estudiante antes de comenzar la actividad de comprensión oral porque estos factores condicionan la manera en que el oyente desarrolla los procesos de comprensión oral y su actuación" $(2009,14)$.

${ }^{6}$ También recogen este detrimento en el tiempo de clase empleado en pro de la CA autores como Feyten (1991) y Harlow y Muyskens (1994).

${ }^{7}$ No deja de resultar paradójico que se destine un tiempo elevado a la conversación dejando de lado la comprensión auditiva máxime si tenemos en cuenta que difícilmente vamos a 
tomamos como base lo señalado hasta ahora. Canale, por ejemplo, defiende que

hay una etapa de comprensión que debe preceder a la etapa de producción en la enseñanza de la segunda lengua (...) en las etapas iniciales del aprendizaje de la segunda lengua quizás debería darse más importancia a las actividades de audición y lectura orientadas al conocimiento y a la habilidad que a aquellas dedicadas a la expresión oral y a la escritura. $(1995,74)$

\section{DISCURSOS ORALES TRADICIONALES}

La comprensión auditiva involucra una serie de aspectos que van desde lo más sencillo, o sea la comprensión del fonema, hasta otros aspectos paralingüísticos más complejos como el significado de lo que se está escuchando, además de la entonación, el énfasis y la velocidad con que se enuncia el mensaje, por ejemplo,

para lograr escuchar se deben interpretar sonidos producidos oralmente, lo que a su vez, implica que esta tiene que distinguir los fonemas de la lengua, o sea las unidades más pequeñas del idioma. Es esto lo que permite a la persona saber que, cuando escucha alguna expresión, ésta se manifiesta en una lengua y no en otra. (Córdoba, Coto y Ramírez, 2005, 3)

Hablar de fonética en la red $^{8}$, es mencionar el proyecto desarrollado por la Universidad de Iowa, Fonética, los sonidos en Internet ${ }^{9}$. En esta, en apariencia, modesta página encontramos aspectos fonéticos como el modo, el lugar y la voz. Con gran detallismo ${ }^{10}$, nos va desgranando los sonidos según sus grados distintivos. Cuenta, además, con una imagen interactiva en la que se pormenorizan los movimientos y los órganos que intervienen en la producción del sonido.

poder comunicarnos si no entendemos el mensaje que quiere transmitirnos nuestro interlocutor.

${ }^{8}$ Recordemos que la comprensión oral es un camino de doble dirección en todo proceso conversacional.

${ }^{9} \mathrm{http}: / /$ www.uiowa.edu/ acadtech/phonetics/spanish/frameset.html

${ }^{10}$ Es necesario poseer conocimientos básicos de fonética. 
Menos ambicioso, pero no por ello menos pragmático, es el trabajo desarrollado por Aurora Patrick. Con el membrete de Español para extranjeros ${ }^{11}$ esta profesora comparte un gran cúmulo de actividades sin caer en oscurantismos científicos. El apartado destinado a la fonética carece de explicación teórica. Se elige la letra (vocal o consonante) y aparece un listado de palabras con un dibujo de apoyo. Se acompaña de aplicación didáctica con ejercicios autocorregibles. Las actividades destinadas a reconocer el sonido y discernir entre las palabras son aptas y atractivas para realizarlas en una pizarra digital. La página se presenta, realmente, como un ejercicio básico de reconocimiento y asociación de sonidos y letras.

Forvo ${ }^{12}$, autodenominada como: "la guía de pronunciación jamás vista"; nos muestra, basándose en trabajo colaborativo digital, palabras pronunciadas por diferentes hablantes, todos reales. Usuarios de todo el mundo graban el audio de la palabra y el alumno o el profesor pueden elegir qué pronunciación escoger eligiendo la ciudad de origen de la voz. Es una buena opción para discriminar entre las distintas pronunciaciones y acentos dentro de un idioma -la página no es de uso exclusivo de la lengua castellana -todas las variedades hispánicas. Cada palabra se acompaña de un mapa donde se nos señalan los países de origen de los hablantes que han grabado dicho término. Permite descargar los audios en mp3 y admite, asimismo, votar la calidad de la pronunciación del vocablo.

El trabajo oral y auditivo de la pronunciación y reconocimiento de palabras es una labor necesaria en el aula de idiomas pero no siempre reconocer un vocablo lleva consigo entenderlo. El alumno se apoya en el contexto para poder deducir el significado de los términos que no conoce y así poder acceder al significado global del texto.

Los materiales destinados a la consecución de la destreza auditiva en el aprendizaje de lenguas están siempre rodeados de una cierta artificialidad. Se intenta representar el lenguaje oral real pero los discursos se convierten en parientes pobres de la rica y auténtica conversación verdadera. Todo ejercicio destinado a ejercitar esta habilidad comunicativa no deja de ser una impostura.

$\mathrm{Si}$ a los fingidos actos de habla les añadimos las características típicas (según recoge el DTC) de estos recursos adulterados nos encontramos con: una vocalización forzada, una pronunciación estándar no dialectal, el ritmo discursivo es lento y uniforme, la entonación se desarrolla con oscilaciones exageradas, el vocabulario está controlado según el nivel de los alumnos, las oraciones completas y adecuadas, también, a su nivel de gramática y la aparición de repeticiones abusivas de determinadas formas lingüísticas.

Los materiales sonoros en el aprendizaje de idiomas se sitúan en un punto bastante alejado del lenguaje real y atesoran pocos puntos en común.

\footnotetext{
${ }^{11} \mathrm{http}: / /$ www.aurora.patrick-nieto.fr/index.html

$12 \mathrm{http}: / /$ es.forvo.com
} 
La pregunta que debe plantearse es qué siente el alumno cuando se enfrenta de lleno a un mundo inentendible y gobernado por unas normas completamente alejadas de las que instauran los audios trabajados en el aula.

Se mantiene aquí la tendencia didáctica de los años sesenta y setenta, tratada por Asher (1965 y 1972) y Postovsky (1974 y 1981), cuando se creía que el aprendizaje de segundas lenguas se alcanzaba al poner al alumno en contacto, durante un largo tiempo, con unidades fraseológicas estándares para que, mediante su repetición, pudiera llegar mantener una conversación en el idioma aprendido.

Partiendo de estas premisas cognoscitivas y didácticas, se deben evaluar los recursos que pueblan la web, océano en el que como reconocía Piñol (2002) se puede tanto navegar como naufragar, "hay que comenzar diciendo que en la red es muy fácil encontrar recursos para aprender (...). Otra cuestión sería analizar si realmente esos recursos cumplen su objetivo, (...) y también si aprovechan todas las posibilidades que brinda Internet" (Piñol, 2004, 171).

La serie de páginas, que se muestran a continuación, no pretenden ser un corpus definitivo pero sí que engloban los perfiles de tratamiento de la CA en Internet y nos sirven para elaborar una idea de cómo se presenta el panorama dentro de la explotación y el desarrollo de esta destreza comunicativa.

En Netlanguages ${ }^{13}$, tras realizar un registro, se puede disfrutar de forma gratuita de una unidad al mes; los otros servicios son de pago. Sigue el esquema tradicional de audios creados ex profeso para el aula de idiomas. Los contenidos no son reales. Cuenta con un índice esquemático en el que se desglosa la información léxica y gramatical que vamos a tratar. Se añade un enlace para poder disponer de los materiales en pdf. Aquí, además, de encontrar la transcripción del audio se dan actividades para ejercitar la parte gramatical y léxica. El audio puede descargarse en mp3 para todo tipo de usuario, tanto para los de iTunes como para los que utilicen otros programas de reproducción de podcast. Todo ello, solamente, en la unidad gratuita, no hay que olvidar que es un curso en el que hay que matricularse.

En Vídeo $E L E^{I 4}$, se encuentran vídeos para aprender español organizados por niveles y estructuras gramaticales. La gramática es muy breve. La mayoría de los vídeos carecen de las guías didácticas y de sus soluciones. Los vídeos están subtitulados, un hecho que facilita enormemente la comprensión auditiva y que ayuda al alumno a la consecución del entendimiento. La duración es excesiva, por ejemplo, superan los cinco minutos en el nivel B2 y cuatro minutos y medio en el $\mathrm{B} 1{ }^{15}$. Las estructuras gramaticales que aparecen son las básicas para cada situación,

\footnotetext{
${ }^{13}$ Empresa fundada en 1998 perteneciente al conocido grupo International House The IHLS Group. http://www.netlanguages.com/info/english/individual/index.php\#resources

${ }^{14} \mathrm{http}: / /$ www.videoele.com

${ }^{15}$ En una conversación real, en cuatro minutos, un hablante puede producir en torno a mil seiscientas palabras.
} 
funcionan como segmentos estándares en la conversación. Como reconoce Martín Peris (1991) la práctica no debe basarse en un mero ejercicio de repetición y comprensión de estructuras modelo, sino que se deben interpretar mensajes nuevos que pueden y tienen que contener contenidos inéditos no aprendidos con anterioridad, tal y como sucede en la vida real.

La página Recursos DELE ${ }^{16}$ mantiene el enfoque tradicional del uso del discurso oral en el aula. La locutora habla de forma pausada remarcando la pronunciación de manera extremadamente forzada, no es nada natural, resulta sobreactuado, tiene una apariencia robótica. La falsedad no tiene nada que ver con la sencillez. No podemos "disfrutar" en estas líneas del sonido del discurso pero mantiene los preceptos sagrados de la oralidad más tradicional, lentitud, pronunciación forzosa, silabeo de palabras hasta el aburrimiento, artificio y falsedad en la oralidad.

Note in Spanish ${ }^{17}$ es una página destinada a anglófonos. Los discursos orales creados específicamente para desarrollar la destreza auditiva son presentados y explicados en inglés. El audio vuelve a recaer en la lentitud de la pronunciación forzando las sílabas y estirándolas. Es necesario matricularse para disponer de las explotaciones didácticas de los audios.

Fluency Prof $^{18}$ también está pensada para hablantes ingleses. Los materiales sonoros, muy variados, se clasifican en: mitos y leyendas, fábulas, diálogos, eventos culturales y frases y vocabulario. Aunque los audios no son reales se intenta variar los dialectos de los locutores para tener así un aspecto más amplio de la heterogénea pronunciación del español. Busca aproximarse a las hablas reales. Los ejercicios que acompañan a las grabaciones son autocorregibles. Aunque intenta imitar la realidad, la pronunciación forzada pone en evidencia el origen irreal de estas grabaciones.

La Familia García Romero ${ }^{19}$, es un proyecto desarrollado por la Fundación de la Lengua y la Universidad de Valladolid. Destinado al nivel A1 del Plan Curricular del Instituto Cervantes (PCIC) cuenta con un apartado denominado 'Una escena familiar' donde se explota el ejercitamiento de esta destreza. Es totalmente gratuito pero hay que darse de alta en la página para poder acceder a los contenidos. Los materiales han sido creados ex profeso para la enseñanza y el aprendizaje del español; los diálogos son pausados pero intentan imitar las conversaciones reales, no se alargan ni fuerzan las sílabas. Es un material muy apetecible y amable. Viene acompañado por ejercicios que refuerzan las otras destrezas y por breves cuadros gramaticales.

\footnotetext{
${ }^{16} \mathrm{http}: / /$ www.recursosdele.com

${ }^{17} \mathrm{http}: / /$ www.notesinspanish.com

${ }^{18} \mathrm{http}: / / \mathrm{www}$.fluencyprof.com/free-spanish-audio.html

${ }^{19} \mathrm{http}$ //www.fundacionlengua.com/garcia-romero/es/material-didactico/art/2367/
} 
Lingus $T V^{20}$ es un curso en línea formado por vídeos destinados, nuevamente, a anglófonos. Cada vídeo viene acompañado por unas breves notas gramaticales: un simple listado de estructuras tipo con sus equivalentes en inglés. Mantiene la división antigua de 'inicial', 'intermedio' y 'avanzado'. La duración no supera los dos minutos. Los diálogos intentan ser reales y utilizan multitud de frases y expresiones hechas. El alumno ha de saber 'pillar' las implicaturas y los dobles sentidos. A pesar de no ser un material real se aproxima mucho a lo que un discurso natural debe ser.

\section{LA COMPRENSIÓN AUDITIVA DE HABLAS REALES}

De entre el maremágnum de audios disponibles en la red y no destinados a priori al aprendizaje de idiomas, se han seleccionado aquellas herramientas que parecen mejores por su valor pragmático y por su posibilidad de explotación en el aula.

Martín Peris (1991) y Maite Cabello (1998) reclaman la necesidad de emplear textos reales auténticos en los procesos de comprensión auditiva, textos grabados en la vida cotidiana alejados de las anquilosadas, repetitivas y pausadas grabaciones enlatadas de los manuales al uso. Estos textos poseen los rasgos peculiares del lenguaje oral y se apoyan además en los contextos situacionales necesarios para que la comprensión sea efectiva.

Comenzamos por una página destinada a las audioguías, Audioviator ${ }^{21}$. Recoge multitud de explicaciones de museos, obras de arte, ciudades...El discurso no va demasiado rápido y puede ser explotado dentro de la clase de español. Permite que el docente muestre, además de la enseñanza lingüística derivada de la escucha, conocimientos culturales de obligado tratamiento en el aula de ELE. Además cuenta con la posibilidad de que sea el usuario el que elabore las guías, hecho que funciona muy bien en el aula, ya que los propios alumnos podrán trabajar otra serie de competencias como las orales y analizar el trabajo de sus compañeros.

De nueva hornada es el portal Eleclips ${ }^{22}$, que cuenta con un repositorio de casi de 5000 vídeos que recogen todas las variedades del español, tanto el peninsular como el de Hispanoamérica. La inclusión de material autentico de los países de habla hispana es más que una necesidad debido, principalmente a la riqueza cultural y variación dialectal del mundo lingüístico del español (Bronch-Bruevich 2006). Los vídeos de breve duración, que va aumentando según el nivel sea más o menos alto, van acompañados de la transcripción, de las pertinentes explicaciones gramaticales y de los correspondientes ejercicios para trabajar todos los aspectos recogidos en el PCIC: aspectos gramaticales, funciones comunicativas, pronunciación y prosodia, nociones específicas, referentes culturales y saberes y comportamientos socioculturales. Los ejercicios son autocorregibles y algunos se

\footnotetext{
${ }^{20}$ http://www.lingus.tv/

${ }^{21} \mathrm{http}: / / \mathrm{www}$.audioviator.com/es/

${ }^{22} \mathrm{http}: / /$ eleclips.agilicedigital.com
} 
acompañan de sugerencias para trabajar en clase. Es un material que utiliza audios reales y que se ajusta fielmente a los cánones y patrones marcados en el PCIC. El material con el que trabajan es de gran calidad. El portal exige del pago de una cuota anual para poder acceder a todos sus recursos.

El archivo de la experiencia ${ }^{23}$ es un portal destinado a escuchar a nuestros mayores,

recoge el relato de personas [...] en distintos lugares de España. Hombres y mujeres cuentan ante la cámara una experiencia, un recuerdo o una anécdota que forma parte de sus vidas y que sirve para explicar a las generaciones más jóvenes la historia reciente de nuestro país. Cada protagonista complementa su testimonio con un objeto, un documento o una fotografía que ilustrará su relato. (El archivo de la experiencia)

Es un material en bruto con audios reales ${ }^{24}$ que pueden ser trabajados y explotados en el aula de español. Las posibilidades didácticas que se nos abren son muchas. La dicción es pausada, llena de explicaciones; las anécdotas son interesantes. Es un material muy amable y recomendado, lleno de historias de vida y experiencias personales.

En este portal los contenidos se dividen en dos grandes bloques: 'Testimonios' y 'Monográficos' y versan en torno a los siguientes núcleos temáticos: cultura, deportes, economía, acontecimientos y sociedad. Ninguno de los vídeos supera los tres minutos.

Banco de textos ${ }^{25}$ es un portal destinado a las actividades basadas en textos literarios, posee un apartado de audio-lecturas ${ }^{26}$ donde usuarios de la página, de edad escolar, dejan la grabación de un fragmento de una obra literaria, los oyentes o lectores sucesivos pueden volver a grabar ese mismo texto, así se va formando una biblioteca de archivos de audio digitales con diferentes acentos, entonaciones... Es un proyecto 2.0 que resalta el valor intrínseco del texto escrito y lo convierte en su único protagonista para mejorar y fomentar la lectura. Es un proyecto común de alumnos de educación primaria y secundaria, de docentes y de usuarios, todos ellos aportan los materiales. Al estar pensado para nativos carece de esa artificialidad y reiteración de los audios tradicionales destinados a la CA.

\footnotetext{
${ }^{23} \mathrm{http}: / /$ www.archivodelaexperiencia.es/

${ }^{24}$ El proyecto, que se realizó en toda la geografía española, tenía como fin "recoger los relatos de personas que han querido compartir sus recuerdos" (el archivo de la experiencia) y se realizó durante el bienio 2007-2009 amparado por el Ministerio de Industria, Turismo y Comercio y con ayuda del Fondo Europeo de Desarrollo Regional.

${ }^{25} \mathrm{http}$ ://recursosdidacticos.es/textos/listado_actividades.php

${ }^{26} \mathrm{http}: / /$ recursosdidacticos.es/textos/listado_audiolecturas.php
} 
Zambombazo ${ }^{27}$ es un blog autodefinido como "una explosión de lengua y cultura". Ofrece una "variada oferta de actividades divertidas basadas en obras culturales (canciones, tiras cómicas, carteles de cine, publicidad, arte, etc.) en las que se pretende aprender por descubrimiento sobre el idioma español y las culturas del mundo hispanohablante". Hay un apartado denominado 'Entrevistas' en el que se ponen a disposición del usuario una gran cantidad de discursos orales en bruto, sin aplicación didáctica ni tratamiento alguno, material que debe trabajarse antes de ser usado en el aula.

Audiria $^{28}$ es otro de los ejemplos de una CA mal entendida. Toma como base vídeos de youtube que el alumno ha de visionar pero luego se trabajan otras destrezas como las gramaticales y la comprensión lectora ya que los ejercicios que acompañan a cada vídeo están relacionados con la transcripción del mismo, no con la comprensión de su audio.

Ver-Taal ${ }^{29}$, posee un apartado denominado 'Ejercicios de escucha' con un gran grupo de vídeos extraídos de telediarios y acompañados de preguntas de comprensión auditiva. Utilizan material real, los ejercicios se autocorrigen y el alumno puede ver si ha entendido lo que acaba de escuchar y ver. Ninguno de los vídeos supera los dos minutos de duración.

Spanish podcast ${ }^{30}$ es una colección de textos de situaciones de comunicación real de la vida cotidiana estructurados como diálogos. En cada episodio nos encontramos con vocabulario, expresiones coloquiales y su correspondiente apoyo gramatical. Aunque se conciba como material didáctico, las grabaciones son excesivamente largas y es un material que debe ser analizado y trabajado fuera del aula antes de probarlo dentro.

Practica Español ${ }^{31}$ es una página que, basándose en las noticias de la Agencia EFE, desarrolla ejercicios de comprensión auditiva. Avalan este portal la Fundación de la Lengua Española y el Instituto Cervantes. Mantiene la división en los seis niveles del PCIC y cada audio viene clasificado en el nivel correspondiente. Actualizan todos los días sus archivos y puedes encontrarte, incluso, noticias de la misma mañana.

La editorial Santillana y la Cadena Ser han elaborado una serie de materiales destinados al desarrollo de la destreza auditiva. En Documentos radiofónicos ${ }^{32}$ nos encontramos con audios reales de programas de radio con temas de actualidad acompañados de actividades de comprensión. El único aspecto negativo que se le podría achacar a este proyecto es la excesiva duración de los audios.

\footnotetext{
${ }^{27}$ http://zachary-jones.com/zambombazo/

${ }^{28} \mathrm{http}: / / \mathrm{www}$.audiria.com

${ }^{29} \mathrm{http}: / / \mathrm{www} . v e r-$ taal.com

${ }^{30} \mathrm{http}: / / w w w . s p a n i s h p o d c a s t . o r g /$

${ }^{31} \mathrm{http}: / /$ www.practicaespanol.com

${ }^{32} \mathrm{http} / /$ www.santillanaele.com/web/index.php?doc_radiofonicos_es
} 
Spanish proficiency exercises ${ }^{33}$ de la Universidad de Texas es una página creada para el aprendizaje del español, está compuesta por un nutrido número de vídeos, grabados por hablantes nativos no profesionales y dividida en niveles de aprendizaje. En cada nivel podemos encontrar en torno a 15 vídeos clasificados temáticamente. Los vídeos son bastante breves, con una duración de alrededor de medio minuto. No existe explotación didáctica, simplemente se accede al vídeo y se visiona con la transcripción en español, en inglés o sin ningún apoyo textual que lo acompañe.

Existe mucha más oferta sonora de la que aquí hemos reseñado. Sirvan estos ejemplos como paradigmas de los materiales que puede ofrecernos la red. ${ }^{34}$

\section{APLICACIONES DIDÁCTICAS}

Todos los materiales reales al no haber sido creados con una finalidad didáctica han de ser trabajados por el docente antes de su manejo en el aula. Hay que tener en cuenta los siguientes criterios a la hora de elegir y trabajar los audios extraídos de la red:

- La calidad del audio. Las grabaciones reales que no han sido creadas para un fin específico (una conversación entre amigos, una entrevista en un mercado o en la calle, un testimonio...) puede que no dispongan de un audio demasiado claro; es posible que el ruido ambiental dificulte la comprensión. Se intentará en la medida de lo posible que el audio tenga una calidad mínima. Por muy interesante que sea la grabación si se escucha con dificultad no es apta para nuestro fin, el uso no será efectivo para la consecución de la CA.

- La claridad del audio. Es factible que la calidad del sonido sea perfecta pero que las personas que hablan no lo hagan de manera adecuada (una mala pronunciación, una velocidad excesiva, la superposición de discursos de varios hablantes...). Si el hablante nativo experimenta dificultad a la hora de comprender no debería emplearse esa grabación para el aula.

- La duración es otro de los elementos importantes a la hora de explotar didácticamente un recurso auditivo. El tiempo de la audición, que irá aumentando según el nivel tratado, no deberá ser excesivamente largo. A la dificultad intrínseca de un audio real no se le debe añadir un exceso de contenidos. Si fuera necesaria, por algún motivo, la escucha de una audición completa demasiado extensa, se fraccionará y se usará por partes de una longitud más adecuada. Se trabajarán los

\footnotetext{
${ }^{33} \mathrm{http}: / /$ www.laits.utexas.edu/spe/index.html

${ }^{34}$ Somos conscientes de que hemos dejado sin reseñar los estudios de corpus orales de aprendientes de español lengua extranjera que se están realizando desde las universidades y que tienen también como una de sus finalidades la implementación de materiales didácticos y el uso directo por parte de los estudiantes. En ese sentido destacan las tesis doctorales dirigidas por el profesor Antonio Moreno Sandoval desde la Universidad Autónoma de Madrid. Véase también Campillo Llanos et al. (2010).
} 
miniaudios de manera independiente, pudiéndose al final, hacer una escucha completa y un trabajo final más global. Este hecho, aunque aleja, evidentemente, el intercambio comunicativo de las conversaciones reales, parece bastante útil, puesto que el alumno realiza actividades de concentración breves, que le permiten no cansarse mentalmente y acceder a la mayor información posible. Hacer audiciones cortas no es lo mismo que hacer escuchas pausadas y lentas.

Los tiempos estimados, para una dificultad de contenido normal, serían: veinte a treinta segundos para $\mathrm{A} 1$; de treinta a cuarenta para $\mathrm{A} 2$; en torno a un minuto para $\mathrm{B} 1$; y de minuto y medio a dos minutos para B2. En los niveles superiores $(\mathrm{C} 1 \mathrm{y}$ C2) la duración de la grabación dependerá de la cordura del docente. La dificultad de estos niveles no se encuentra en la longitud de los audios sino en la captación rápida de los componentes pragmáticos, los dobles sentidos y las inferencias. No se recomienda utilizar audios mayores de tres minutos.

- El contenido temático del audio. El grado de dificultad nunca debe ser el único criterio a adoptar. Cualquier audio que pueda suscitar el interés de nuestros alumnos será siempre un audio adecuado. Hay que buscar una grabación cuyo contenido sea ameno y atractivo para el discente. No conviene crear un aula aburrida; la abulia es enemiga del aprendizaje. Pueden ser usados audios de temas culturales para cubrir así el aprendizaje de contenidos socioculturales tan necesarios en la adquisición de toda competencia lingüística. En un aula multicultural se deben evitar temas conflictivos: religión, creencia, identidad sexual, derechos y libertades... En definitiva, puede emplearse cualquier tipo de audio o vídeo que, manteniendo los principios básicos de cortesía, sea atractivo y resulte interesante para nuestros alumnos.

- El contenido gramatical del audio no debe obviarse ni tampoco debe ser considerado como un lastre para el docente. El vocabulario debe estar ajustado (más o menos) al nivel, así como las estructuras gramaticales básicas. Pero ha de imperar siempre la lógica del profesor. Si el modelo de comprensión auditiva empleado por el alumno engloba procedimientos analíticos y sintéticos, se apoyará en el contexto cuando deba enfrente a un vacío cognoscitivo y tenga que realizar la descodificación.

Si tenemos en cuenta las actividades didácticas que podemos realizar con una audición extraída de Internet, además de las típicas preguntas de comprensión oral, podemos además tener presentes las tipologías de Richards $(1983,235)$ o de Giovannini, Martín Peris, Rodríguez y Simón (1996, 12-13) o Cubillo, Coto y Ramírez $(2005,12-16)$ entre otros. Así podríamos didactizar un vídeo mediante las siguientes actividades:

- $\quad$ Actividades de relación o de distinción: seleccionar entre varias la respuesta correcta, aquella que encaja con el texto escuchado.

- Actividades de transferencia: los alumnos representan en un dibujo la información derivada de la escucha. Este tipo de actividad es muy útil para, por ejemplo, los adjetivos y la descripción, o para seguir unas normas. 
- Actividades de registro: son aquellas en las que el alumno ha de rastrear un dato (o varios) o una información concisa (o varias) en el texto escuchado.

- Actividades de ampliación: añadir al relato elementos que no tenía: un título, una continuación, un previo...

- Actividades de condensación: resumir lo escuchado, crear un listado de ideas que engloben lo que el alumno acaba de escuchar.

- Actividades de respuesta: responder a las preguntas de comprensión del audio. Estas preguntas no tienen porqué ser solamente textuales, sino que también pueden realizarse preguntas que obliguen a hacer inferencias, que impliquen una deducción, cuestiones sobre detalles muy concretos o, incluso, puede solicitarse al alumno que evalúe o reaccione ante lo que ha escuchado.

- Actividades de ordenación: ordenar por orden de escucha una serie de datos recibidos previamente.

- Actividades de predicción: consiste en predecir qué acontecimientos se van a desarrollar después en la historia o el diálogo escuchado.

- Actividades de transcripción: escuchar y escribir lo escuchado. Aunque Davis y Rinvolucri (1988) desplieguen todo un decálogo en defensa del uso del dictado en el aula y en los laboratorios lingüísticos, hay que señalar que el dictado retarda el ritmo de las clases, llevándolas indefectiblemente a la abulia y al anquilosamiento más rancio de la antigua docencia. Por mucho que se preocupe en defender esta nueva metodología basada en "un viejo tipo de ejercicio" no consigue hacerlo atractivo.

La gama de actividades, que podemos aplicar a un recurso sonoro para desarrollar la destreza auditiva, es tremendamente amplia. Será el docente el que elija cuál es el tipo de ejercicio más pertinente en cada caso, dependiendo del fin buscado.

La transcripción de todo audio sí debería ser obligatoria para realizar un trabajo final de escucha en el que el alumno pueda a la vez leer lo que está escuchando, así muchas de las dudas, vacíos sonoros, problemas, etc., encuentran la solución de una manera visual. La lectura contribuye también al asentamiento de estructuras gramaticales.

\section{CONCLUSIONES}

La necesidad de utilizar discursos reales en el desarrollo de la competencia auditiva es fundamental si queremos que los estudiantes adquieran un conocimiento preciso de la lengua meta, y consigan una comprensión auditiva efectiva, de cara a verse inmersos en una situación real de uso de la lengua. Como hemos visto, la existencia de material excesivamente sobreactuado, o textos 
construidos sobre situaciones antinaturalistas, no favorecen en absoluto el aprendizaje.

Empleando material real, o lo más natural posible, conseguiremos que el alumno:

- Se sienta confiado a la hora de demostrar sus conocimientos, sabiendo que los textos con los que está trabajando pertenecen realmente a la vida cotidiana del país de acogida y no son, simplemente, diálogos adaptados a su nivel. Para él supone un reto el enfrentarse a estos discursos, pero descubre que sus capacidades lingüísticas son suficientes para poder entenderlos.

- Sea capaz, con el tiempo, la práctica y la adaptación del oído a los materiales reales, de descifrar y de comprender oralmente las noticias de un telediario, los programas radiofónicos u otros audios considerados como complicados, lo que le dará ánimos para seguir enfrentándose a los diferentes retos que la lengua le propone en su andar diario.

- Aprenda las palabras exactas pertenecientes al tema estudiado; y no mediante la adaptación del vocabulario, o la simplificación de la sintaxis, aprende en directo, en el uso mismo y real de la lengua española.

- Pueda además, a través de los materiales reales expresarse siguiendo las pautas asimiladas, utilizando el vocabulario específico y asentando los argumentos desarrollados en las conversaciones cotidianas.

- Asimismo, desarrolle aspectos lingüísticos que vayan más allá de los meramente morfosintácticos o semánticos, utilizando o aprendiendo a utilizar, y familiarizándose con los aspectos coloquiales de la lengua: juegos de palabras, dobles sentidos, inferencias, metáforas...

\section{BIBLIOGRAFÍA}

ASHER, J. J. (1965): "The Strategy of Total Physical Response: An Application to Learning Russian", en International Review of Applied Linguistics 3, 291-300.

ASHER, J. J. (1972): “Children's First Language as a Model for Second Language Learning", en Modern Language Journal 56, 133-39.

BRONCH-BRUEVICH, X. (2006): "Prácticas actuales y direcciones futuras para la enseñanza de la comprensión auditiva a los hablantes de inglés en el ambiente multimedia", en redELE, 7, s.p. Disponible en Web: http://goo.gl/hYFBBu

CABELLO, M. (1998): La comprensión auditiva en ELE, Madrid, Universidad Antonio de Nebrija.

CAMPILlO LLANOS, L., GONZALO GÓMEZ, P., GUIRAO MIRAS, J.M MORENO SANDOVAL, A. (2010): Español oral en contexto, Vol.1. Textos de español oral. Material de ELE basado en corpus. Comprensión auditiva. Madrid. Servicio de publicaciones de la Universidad Autónoma de Madrid.

CANALE, M. (1995): "De la competencia comunicativa a la pedagogía comunicativa del lenguaje", en Competencia comunicativa. Documentos 
básicos en la enseñanza de lenguas extranjeras, LLOBERA, M. et al., Madrid, Edelsa, 63-81.

CASAÑ NÚÑEZ, J. C. (2009): Didáctica de las grabaciones audiovisuales para desarrollar la comprensión oral de lenguas extranjeras, Suplementos marcoELE 9. Disponible en Web: http://goo.gl/D2ihYD

COOK, V. (2004): Second Language learning and language teaching, Londres, Hodder Education.

CÓRDOBA, P., COTO, R. Y RAMÍREZ, M. (2005): "La enseñanza del inglés en Costa Rica y la destreza auditiva en el aula desde una perspectiva histórica", en Actualidades Investigativas en Educación, 5(2), 1-12. Disponible en Web: http://goo.gl/p4MJod

CUBILLO, P., COTO KEITH, R. y RAMÍREZ SALAS, M. (2005): "La comprensión auditiva: definición, importancia, características, procesos, materiales y actividades", en Revista Electrónica Actualidades Investigativas en Educación, 5.1, 1-17. Disponible en Web: http://goo.gl/rr00h2

DAVIS, P. y RINVOLUCRI, M. (1988): Dictation, Cambridge, Cambridge University Press, 1988.

DUNKEL, P. (1991): "Listening in the Native and Second / Foreign Language: Toward an Integration of Research and Practice" en TESOL Quarterly 25, 43157.

FEYTEN, C.M. (1991): "The Power of Listening Ability: An Overlooked Dimension in Language Acquisition" en Modern Language Journal 75, 173-80.

GIOVANNINI, A., MARTÍN PERIS, E. RODRÍGUEZ, M. Y SIMÓN, T. (1996): Profesor en acción, Madrid, Edelsa.

HARLOW, L. L. y MUYSKENS, J. A. (1994): "Priorities for Intermediate-Level Language Instruction" en Modern Language Journal 78, 141-54.

JAMES, C. J. (1984): "Are you listening: The practical components of listening Comprehension", en Foreing laguage annals, 17.2, 129-134. Tomado de CÓRDOBA, P., COTO, R. Y RAMÍREZ, M. (2005). "La enseñanza del inglés en Costa Rica y la destreza auditiva en el aula desde una perspectiva histórica", en Actualidades Investigativas en Educación, 5(2), 1-12. Disponible en Web: http://goo.gl/p4MJod

KOSTER, C. (1991): "La comprensión oral en una lengua extranjera", en Cable, 8, 5-10.

LONG, D. R. (1987): "Listening Comprehension: Need and Neglect" en Hispania 70, 921-28.

MARTÍN PERIS, E. (1991): "La didáctica de la comprensión auditiva", en Cable, 8, 16-28.

MARTÍN PERIS, E. (Coord.) (2008): Diccionario de términos clave de ELE, Madrid, Instituto Cervantes, SGEL. Disponible en Web: http://goo.gl/Bt8g1

MUELLER, G. A. (1980): "Visual Contextual Cues and Listening Comprehension: An Experiment", en Modern Language Journal 64, 335-40. 
PIÑOL, M. C. (2002): “¿Navegar o naufragar? La WWW y la enseñanza del léxico del español", en Textos. Didáctica de la lengua y la literatura, 31, 53-64.

PIÑOL, M. C. (2004): "La enseñanza de léxico en el aula de español como lengua extranjera. Recursos en Internet para la elaboración de actividades", en Carabela, 56, 171.

POSTOVSKY, V. A. (1974): "Effects of Delay in Oral Practice at the Beginning of Second Language Learning", en Modern Language Journal 58, 229-39.

POSTOVSKY, V. A. (1981): "The Priority of Aural Comprehension in the Language Acquisition Process", en The Comprehension Approach to Foreign Language Instruction, WINITZ, H. (Ed.), Cambridge, Newbury House Publishers, 170-86.

RICHARDS, J. C. (1983): "Listening comprehension: Approach, Design, Procedure", en TESOL Quarterly, vol. 17. 2, 219-240.

RODRÍGUEZ ABELLA, R. M. (2002): "La comprensión auditiva en la enseñanza del español mediante lenguajes específicos", en Testi specialistici e nuovi saperi nelle lingue iberiche. XX Convegno AISP, Vol.II., CUSATO, D. A. y FRATTALE, L., (Coords.), Messina, Andrea Lippolis Editore, 233-244. Disponible en Web: http://cvc.cervantes.es/literatura/aispi/pdf/16/16_231.pdf

POCH OLIVÉ, D. (1988): "Sensación física y realidad mental: de la onda sonora al significado de los enunciados", en Carabela. Monográfico: El desarrollo de la comprensión auditiva en el aula de español como lengua extranjera, 49, 5-15.

ROST, M. (1991): Listening in action, New Jersey, Prentice Hall International.

SPERBER, D. y WILSON, D. (1994): La relevancia: comunicación y procesos cognitivos, Madrid, Visor. 